\title{
Carrier dynamics of Mg-doped indium nitride
}

\author{
H. Ahn*a, C.-C. Hong ${ }^{\mathrm{a}}$, Y.-L. Hong, and S. Gwo ${ }^{\mathrm{b}}$ \\ ${ }^{a}$ Department of Photonics and Institute of Electro-optical Engineering, National Chiao Tung \\ University, Hsinchu 30010, Taiwan, Republic of China; ${ }^{b}$ Department of Physics, National Tsing \\ Hua University, Hsinchu 30013, Taiwan, Republic of China
}

\begin{abstract}
We report the carrier density dependence of carrier dynamics of Mg-doped InN (InN:Mg) films. Recently, we have demonstrated a significant enhancement of terahertz emission from InN:Mg, which is due to the temporal evolution of drift and diffusion currents depending on the background carrier density. We studied the details of carrier dynamics of $\mathrm{InN}: \mathrm{Mg}$ which is crucial for the clarification of the terahertz emission mechanism by performing the time-resolved optical reflectivity measurement on InN:Mg films grown with different Mg-doping levels. Experimental analysis demonstrates that the initial sharp drop and recovery of reflectivity response of InN:Mg films are dominated by photocarrier-dependent bandgap renormalization and band filling processes, whereas the slow decay time constant $\left(\tau_{2}\right)$ of reflectivity of InN:Mg has the strong dependence on the background carrier density. As the carrier density decreases from that of undoped $\mathrm{InN}, \tau_{2}$ of InN:Mg continuously increases and reaches the maximum value at a critical value of $\sim 1 \times 10^{18} \mathrm{~cm}^{-3}$. Interestingly, the strongest terahertz radiation was observed at this carrier density and it keeps decreasing with the increase of carrier density. Intense terahertz radiation corresponds to the fast and large spatial separation of charged carrier density through diffusion and drift. Large spatial separation results in the longer decay time for charged carriers to reach equilibrium after strong emission of terahertz waves, and it explains the similar carrier density dependence of terahertz emission and $\tau_{2}$.
\end{abstract}

Keywords: carrier dynamics, Mg-doped indium nitride, terahertz emission

\section{INTRODUCTION}

Recently, research activities in indium nitride $(\mathrm{InN})$ have been dramatically increased due to its potential applications in high-frequency electronic devices, near-infrared optoelectronics, and high-efficiency solar cells. To realize the InNbased optoelectronic devices, it is essential to have the ability to fabricate both $n$ - and $p$-type InN. Typically, as-grown indium nitride film is $n$-type due to its high electron affinity and its carrier density $(n)$ is in the range of $10^{17}-10^{18} \mathrm{~cm}^{-3}$. $n$-type InN with even higher carrier density is possible by doping $\mathrm{InN}$ with donors such as $\mathrm{Si}$, while the realization of $p$ type InN is known to be very difficult. Although there is still a lack of direct measurement results of hole transport, it has been proposed that the realization of $p$-type InN may be possible by using $\mathrm{Mg}$ as an acceptor dopant. ${ }^{1}$ Meanwhile, due to its narrow bandgap and high electrical mobility, InN has been considered to be a candidate of efficient terahertz emitter. However, due to high intrinsic carrier density, terahertz emission from as-grown $c$-plane InN is typically much smaller than other narrow bandgap semiconductor such as InAs. Recently, we have demonstrated a selective enhancement of terahertz emission from Mg-doped $\mathrm{InN}$ ( $\mathrm{InN}: \mathrm{Mg}$ ). The selective enhancement of emission was due to the competition between drift and diffusion currents depending on the $n$-type carrier density. ${ }^{2}$ Terahertz pulses induced by femtosecond laser pulses have the pulsewidth of $<1 \mathrm{ps,} \mathrm{indicating} \mathrm{that} \mathrm{spatial} \mathrm{redistribution} \mathrm{of} \mathrm{carriers} \mathrm{through} \mathrm{drift} \mathrm{and} \mathrm{diffusion}$ occurs in the same time scale. ${ }^{3}$ Therefore, in order to fully understand the terahertz emission mechanism, it is essential to clarify the details of ultrafast carrier dynamics. There have been several reports on the carrier dynamics of InN films ${ }^{4-12}$ and nanostructures, ${ }^{13,14}$ but no systematic study has been reported on carrier dynamics of InN:Mg and its dependence on background carriers. In order to identify the contribution of background electrons in carrier dynamics of InN, we performed the time-resolved optical reflectivity measurement on InN:Mg films grown with different Mg-doping levels. Experimental analysis demonstrates that bandgap renormalization (BGR) and band filling (BF) processes dominate the initial time dependence of reflectivity. The time constant of hot-carrier cooling in $\mathrm{InN}: \mathrm{Mg}$ was measured to be in the range of subpicosecond to a few picoseconds, which agrees with previous results. ${ }^{4-7,9-11}$

*hyahn@mail.nctu.edu.tw; phone 8863 5712121-56369; fax 88635716631

Ultrafast Phenomena in Semiconductors and Nanostructure Materials XV,

edited by Kong-Thon Tsen,Jin-Joo Song, Markus Betz, Abdulhakem Y. Elezzabi, Proc. of SPIE

Vol. 7937, 79370Y - @ 2011 SPIE · CCC code: 0277-786X/11/\$18 - doi: 10.1117/12.874133

Proc. of SPIE Vol. 7937 79370Y-1 
Meanwhile, the carrier decay time of InN:Mg shows a strong dependence on the background carrier density and its dependence is found to be similar to that of terahertz radiation. In particular, the carrier decay process following the cooling is found to be closely related with terahertz radiation in InN:Mg. Since the terahertz radiation from photoexcited semiconductors is the transient electric dipole radiation due to the acceleration of photoexcited carriers, the amplitude and the polarity of terahertz field are determined by the magnitude and the direction of drift and diffusion current. Strong terahertz emission from InN:Mg is accompanied by the large spatial separation of dipoles and carriers need much longer time to relax to the equilibrium and it explains the correlation between them. In this paper, we also present the details of carrier dynamics of InN:Mg including pump fluence dependence of carrier lifetimes and diffusion coefficient.

\section{EXPERIMENTAL METHOD}

\subsection{Experimental set-up}

The optical pump-probe measurement was performed using a Ti:sapphire laser system, which delivers $\sim 150$ fs optical pulses at a center wavelength of $800 \mathrm{~nm}$. The pump laser beam was incident on the samples at a near-normal angle and the transient reflectivity change $(\Delta R / R)$ of the probe beam incident at $45^{\circ}$ was recorded as a function of the delay time between the pump and probe pulses. Terahertz radiation was measured by using a time-domain terahertz measurement method. In this experiment, the pump laser beam is collimated on the samples with a spot size of $\sim 2 \mathrm{~mm}$ at the incident angle of $70^{\circ}$ and the typical fluences of pump pulses incident at $70^{\circ}$ are in the range of $100-500 \mu \mathrm{J} / \mathrm{cm}^{2}$. Terahertz pulses were detected by the free-space electro-optic sampling method in a 2-mm-thick ZnTe crystal as a function of delay time with respect to the optical pump pulse.

\subsection{Sample preparation}

Wurtzite N-polar, undoped and Mg-doped InN films with different electron concentrations were grown by plasmaassisted molecular beam epitaxy on $\mathrm{Si}(111)$. The film thicknesses of samples are in the range of $1-1.5 \mu \mathrm{m} . \mathrm{Mg}$ doping was performed with a high-purity $\mathrm{Mg}(6 \mathrm{~N})$ Knudsen cell and the Mg doping level was controlled by regulating the cell temperature between 180 and $290^{\circ} \mathrm{C}$. The electron density and mobility of each sample determined by room-temperature Hall effect measurement are subject to the $\mathrm{Mg}$ doping level and their dependence on the $\mathrm{Mg}$ cell temperature is illustrated in Fig. 1(a). Here, the carrier density shows a peculiar dependence on the Mg doping level in such a way that the carrier density first decreases as the $\mathrm{Mg}$ cell temperature increases and then increases at higher $\mathrm{Mg}$ cell temperatures due to longitudinal optical phonon-plasmon coupling. ${ }^{15}$ Figure 1(b) shows the background carrier dependence of MottSchotty plot of C-V data of InN:Mg, which is consistent with previous result. ${ }^{16}$
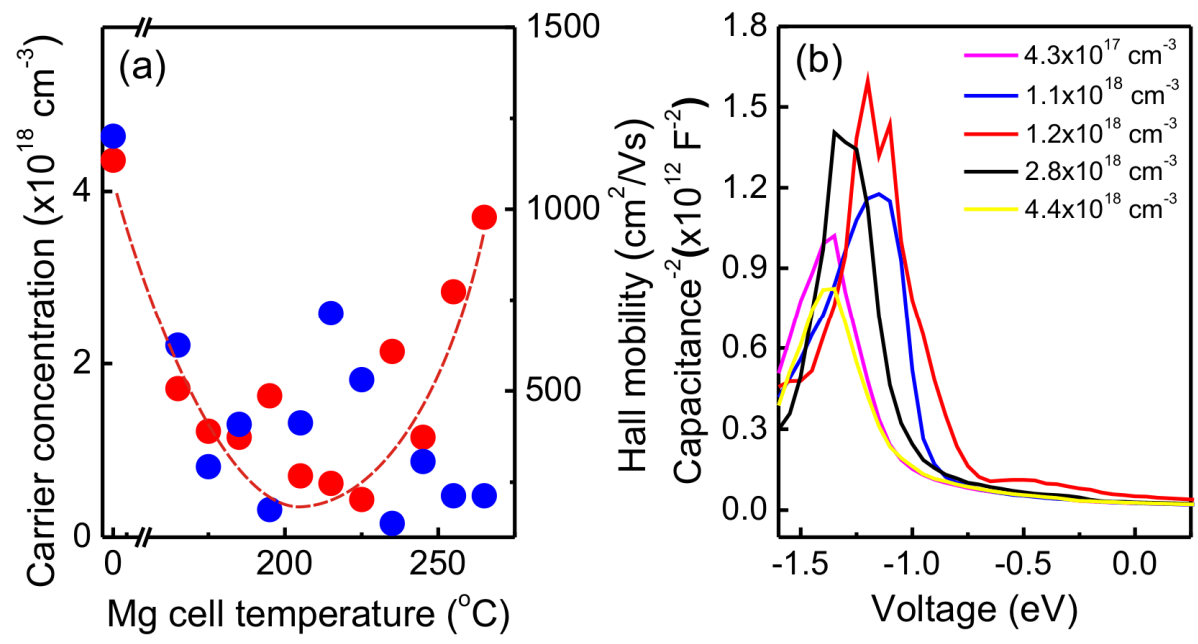

Fig. 1. (a) Background carrier concentration and electron Hall mobility of $\mathrm{InN}: \mathrm{Mg}$ as a function of $\mathrm{Mg}$ cell temperature (b) Mott-Schottky plot of capacitance-voltage data obtained from InN:Mg films using an electrolyte contact. 
We also measured the infrared reflectance of InN:Mg with different background carrier density and plotted it in Fig. 2. The plasma reflection edge shift with respect to undoped $\mathrm{InN}$ film is observed for three InN:Mg films with lower carrier densities.

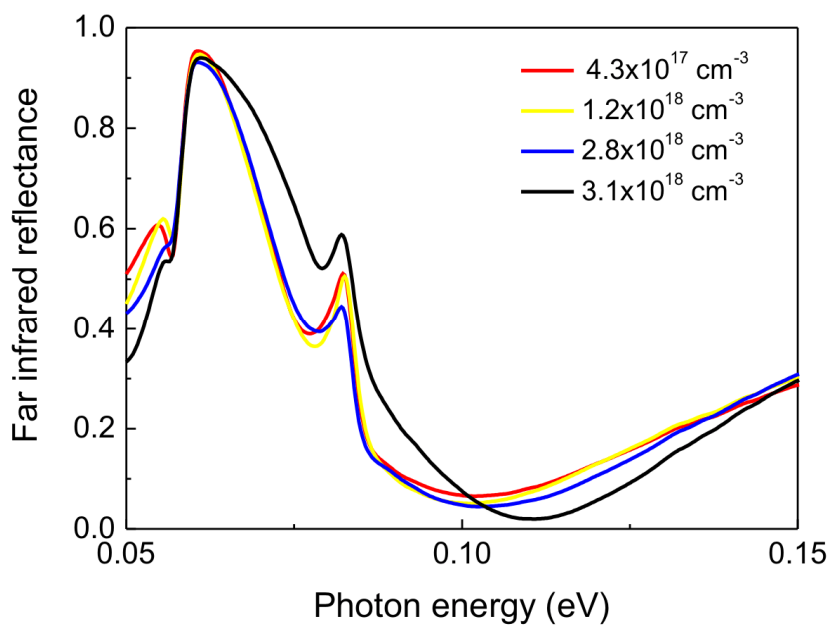

Fig. 2. Infrared reflection curves of $\mathrm{InN}: \mathrm{Mg}$ with background carrier density. The black curve with the carrier density of $3.1 \times 10^{18} \mathrm{~cm}^{-3}$ corresponds to one of undoped $\mathrm{InN}$ film.

\section{ULTRAFAST OPTICAL PUMP-AND-PROBE EXPERIMENT}

Figure 3(a) shows the transient reflectivity of the undoped InN film measured at various excitation fluences $(F)$ ranging from 100 to $500 \mu \mathrm{J} / \mathrm{cm}^{2}$. The differential reflectivity $\Delta R / R$ of the samples excited at $F>200 \mu \mathrm{J} / \mathrm{cm}^{2}$ sharply decreases with the pulsewidth-limited falling time, which is much longer than the carrier-carrier scattering time $(\leq 50 \mathrm{fs})$ measured by the terahertz time-domain spectroscopy method. ${ }^{17}$ The decreased differential reflectivity rapidly recovers to a positive value with a time constant of $\tau_{1}$ and it is followed by a slow decay with a time constant of $\tau_{2}$. The maximum change of reflectivity $-(\Delta R / R)_{\max }$ increases linearly with increasing pump fluence, indicating the contribution of intraband carrier absorption.

For photoexcited semiconductors, the temporal evolution of reflectivity can be affected by the carrier dynamics through several effects, such as BF, BGR, free carrier absorption, and carrier recombination. ${ }^{5}$ Among them, BGR due to the screening of electron-hole Coulomb interaction is the dominant process at high carrier density and its effect causes the decrease of reflectivity. Therefore, the initial sharp decrease of reflectivity observed in Fig. 3(a) indicates the strong contribution of BGR for highly photoexcited InN. As the photocarriers cool down and relax toward the band edge, the BF effect, which increases the reflectivity, begins to be important so that $\Delta R / R$ recovers toward less negative values. At low excitation $\left(F \leq 200 \mu \mathrm{J} / \mathrm{cm}^{2}\right)$, due to the generation of less dense photocarriers, only the $\mathrm{BF}$ effect is observed. The dominant contribution of the BF effect to reflectivity is also observed for InN nanorod arrays which are grown at sample temperature of $520^{\circ} \mathrm{C}$ on $\mathrm{Si}(111) .^{18,19} \mathrm{InN}$ nanorod arrays consisted of nanorods of the diameter of 60 and $130 \mathrm{~nm}$ have higher background carrier density $\left(\sim 10^{19} \mathrm{~cm}^{-3}\right)$ than InN films which exceeds the density of photoexcited carriers at the current pump fluence ${ }^{20}$ experience the strong BF effect and the transient reflectivity sharply increases as soon as the pump pulses arrive. The details of the carrier dynamics of InN nanostructure arrays will be discussed in the future publication.

The carrier lifetimes $\tau_{1}$ and $\tau_{2}$ were obtained from the best fitting to the measured reflectivity using double exponential functions. Figure 4 shows that $\tau_{1}$ increases linearly from $\sim 0.2$ to $1.2 \mathrm{ps}$ as $F$ increases from 100 to 500 $\mu \mathrm{J} / \mathrm{cm}^{2}$, while $\tau_{2}$ is nearly independent on the change of pump fluence. The slow decay of reflectivity can be affected by various processes, such as carrier diffusion, radiative, nonradiative, and Auger recombination. The pump fluenceindependent $\tau_{2}$ in Fig. 4 indicates that the carrier decay mechanism is not affected by radiative or Auger recombination process, but by a process which is independent on the total carrier density, including the densities of background and photoexcited carriers. 

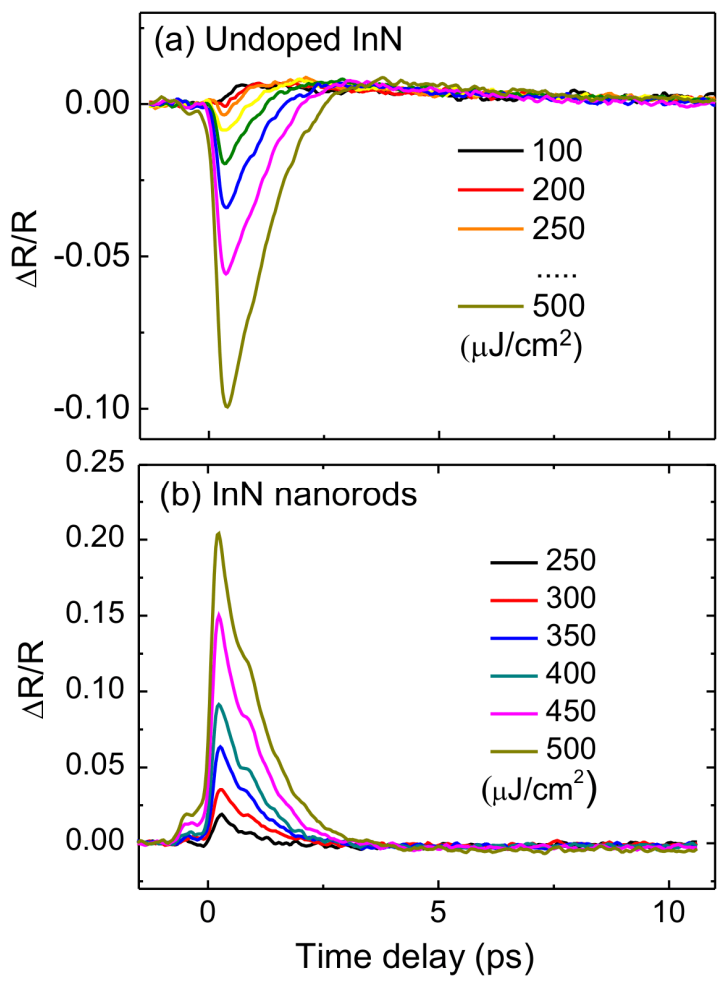

Fig. 3. (a) Reflectivity transient of an undoped InN film with the background electron density of $3.1 \times 10^{18} \mathrm{~cm}^{-3}$ measured under different levels of photoexcitation, (b) Reflectivity transient of a InN nanorod arrays.

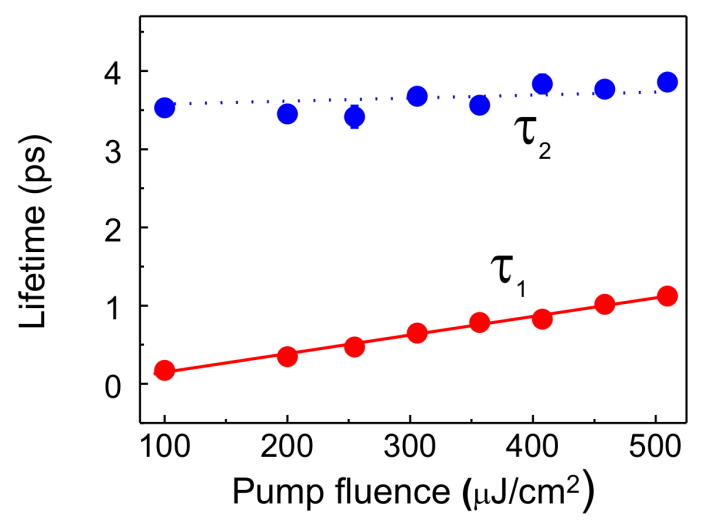

Fig. 4. The carrier recovery $\left(\tau_{1}\right)$ and decay $\left(\tau_{2}\right)$ time constants obtained from the double exponential fits to the reflectivity responses shown in Fig. 3(a).

In order to investigate the roles of the background carrier density in the carrier dynamics in InN, we measured the transient reflectivity of the InN:Mg films with various carrier densities at the pump fluence of $350 \mu \mathrm{J} / \mathrm{cm}^{2}$. Figure 5 shows that while the overall trend of transient reflectivity of InN:Mg is similar to that of undoped InN in Fig. 3(a), each InN:Mg film with different background carrier density has different $\tau_{2}$ and the minimum value of $\Delta R / R$. As it is shown in Fig. $6, \tau_{2}$ has a peculiar dependence on $n$ in such a way that as $n$ decreases from that of undoped $\operatorname{InN}, \tau_{2}$ increases until it reaches its maximum value $\sim 10 \mathrm{ps}$ at $n_{\mathrm{c}} \sim 1 \times 10^{18} \mathrm{~cm}^{-3}$ and decreases as $n$ decreases below $n_{\mathrm{c}}$. Since each InN:Mg film is photoexcited at the same pump fluence, the fast carrier lifetime $\tau_{1}$ corresponding to hot carrier cooling through phonon emission does not show the dependence on the background carrier density $n$. 


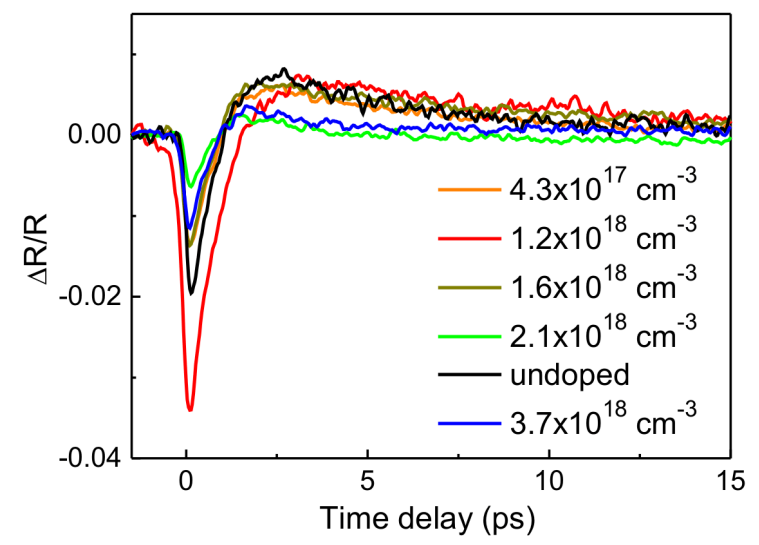

Fig. 5. Transient reflectivity of the Mg-doped InN films with different background carrier densities under the pump fluence of $350 \mu \mathrm{J} / \mathrm{cm}^{2}$.

Interestingly, the intensity of terahertz radiation from the InN:Mg films in Fig. 6(b) shows the similar carrier density dependence to that of decay time. With ultrafast photoexcitation, the spatial redistribution of electrons and holes can occur either by the diffusion field due to the difference in diffusion coefficients for electrons and holes or by the surface accumulation field attributed to the downward band bending by the Fermi level-pinning, whose relations are given by ${ }^{3}$

$$
\frac{\partial \Delta n}{\partial t} \propto G+\frac{\partial}{\partial z}\left[D \frac{\partial \Delta n}{\partial z}\right] \pm \frac{\partial}{\partial z}\left[\mu_{b} E n\right]+\left[\mu_{p h} E \Delta n\right],
$$

where $n(\Delta n)$ and $\mu_{b}\left(\mu_{p h}\right)$ correspond to the carrier density and mobility for background (photoexcited) carriers, respectively, $D$ denotes the diffusion coefficient, and $G$ is the photoexcitation rate. Equation (1) shows that terahertz radiation due to the spatial redistribution of photocarriers are influenced by both background and photoexcited carriers. For highly photoexcited undoped $\mathrm{InN}$ grown along the $c$-plane, terahertz emission is dominated by carrier diffusion (the photo-Dember effect). But the strong Coulomb screening due to its exceptionally high background carrier density makes the terahertz emission from undoped InN small compared to that of other semiconductors, such as InAs. Reduction of the screening of the diffusion field can be achieve by doping InN with $\mathrm{Mg}$ and Fig. 6(b) shows a dramatic enhancement of terahertz emission (at least 500 times higher than that of undoped $\mathrm{InN}$ ) for $\mathrm{InN}: \mathrm{Mg}$ with $n \sim n_{\mathrm{c}} \cdot{ }^{2}$

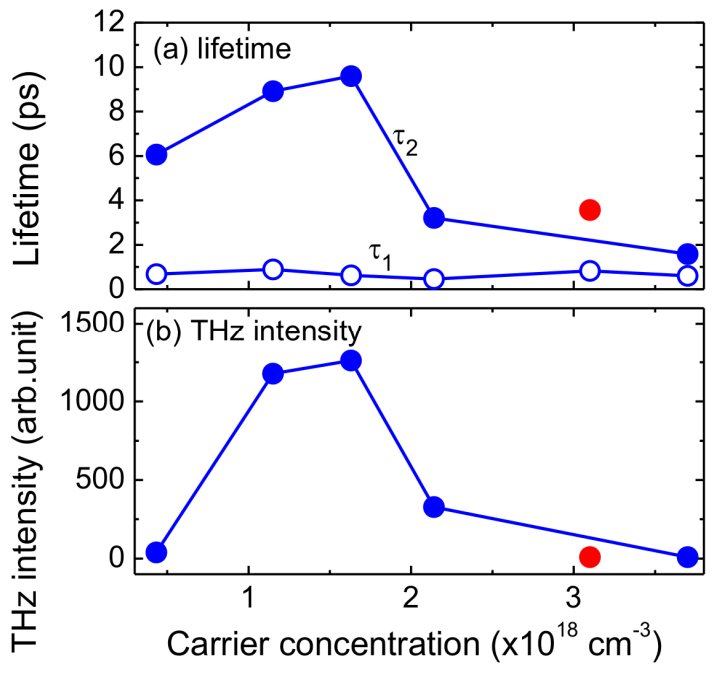

Fig. 6. Carrier density dependence of carrier lifetimes and terahertz radiation intensity of the InN:Mg film. Red circles correspond to those of the reference undoped InN film. 
For InN:Mg with a lower carrier density $\left(n<n_{\mathrm{c}}\right)$, terahertz emission is dominated by drift current which is formed in a region of the near-surface charge depletion layer and flows opposite to the diffusion current. Less intensity of terahertz radiation from InN:Mg with the carrier density $<n_{\mathrm{c}}$ corresponds to less surface band bending within shorter charge depletion layer. $^{2}$

The ultrafast plasma oscillation under diffusion and drift fields gives rise to terahertz waves, which is heavily damped within 1 ps. In such case, intense terahertz radiation can be correlated with fast and large spatial separation of charged carriers. For largely separated electrons and holes, it may take much longer time to recombine to equilibrium after terahertz emission. Therefore, the measured recombination time constant reflects the spatial separation of charged carriers which is determined by the magnitude of terahertz field. And it explains the similar carrier density dependence of the decay time constant and the intensity of terahertz radiation shown in Fig. 6(a) and (b). Finally, we assume the longest decay time constant of $10 \mathrm{ps}$ for InN:Mg to be the time for electrons to diffuse through the penetration depth $(d \sim 133 \mathrm{~nm})$ under the screening-free diffusion field. The estimated longitudinal diffusion coefficient $\left(D_{l} \sim d^{2} / \tau\right)$ is 18 $\mathrm{cm}^{2} / \mathrm{s}$, which is much larger than in-plane diffusion coefficient of $\sim 2 \mathrm{~cm}^{2} / \mathrm{s}$ on the surface measured by time-resolved transient grating spectroscopy.

\section{SUMMARY}

In summary, time-resolved reflectivity measurements have been used to determine the carrier dynamics of Mg-doped InN films. For both undoped and Mg-doped InN films, the initial time dependent behavior of reflectivity is dominated by the interplay between the effects of bandgap renormalization and band filling depending on the density of photocarriers. The slow carrier decay time constant, however, is found to be independent on the pump fluence, in other words, the photocarrier density, but is closely dependent on the background carrier density. In particular, the carrier decay time becomes longest when the background carrier density of InN:Mg is close to the critical density and at the same carrier density, the terahertz radiation from InN:Mg dramatically is enhanced. It indicates that large spatial separation of electrons and holes induces intense terahertz radiation, but it takes much longer time to decay to the equilibrium through the recombination dynamics.

\section{ACKNOWLEDGEMENTS}

This work was supported by the National Science Council (NSC 98-2112-M-009-009-MY3) and the National Nanoscience and Nanotechnology Project (NSC 98-2120-M-007-009) in Taiwan.

\section{REFERENCES}

[1] R. E. Jones, K. M. Yu, S. X. Li, W. Walukiewicz, J. W. Ager, E. E. Haller, H. Lu, and W. J. Schaff, "Evidence for p-type doping of InN, ” Phys. Rev. Lett. 96, 125505 (2006).

[2] H. Ahn, Y.-J. Yeh, Y.-L. Hong, and S. Gwo, "Terahertz emission mechanism of magnesium doped indium nitride," Appl. Phys. Lett. 95, 232104 (2009).

[3] K. Liu, J. Xu, T. Yuan, and X.-C. Zhang, "Terahertz radiation from InAs induced by carrier diffusion and drift," Phys. Rev. B 73, 155330 (2006).

[4] F. Chen, A. N. Cartwright, H. Lu, and W. J. Schaff, "Hole transport and carrier dynamics in InN epilayers," Appl. Phys. Lett. 87, 212104 (2005)

[5] D. Zanato, N. Balkan, B. K. Ridley, G. Hill, and W. J. Schaff, "Hot electron cooling rate via the emission of LOphonons in InN," Semicond. Sci. Technol. 19, 1024 (2004)

[6] J. W. Pomeroy, M. Kuball, H. Lu, and W. J. Schaff, X. Wang, and A. Yoshikawa, "Photon liftetime and phonon decay in InN," Appl. Phys. Lett. 86, 223501 (2005).

[7] K. T. Tsen, J. G. Kiang, D. K. Ferry, H. Lu, and W. J. Schaff, H.-W. Lin, and S. Gwo, "Electron-density dependence of longitudinal-optical phonon lifetime in InN studied by subpicosecond time-resolved Raman spectroscopy," J. Phys. Condens. Matter 19, 236219 (2007).

[8] T.-R. Tsai, C.-F. Chang, and S. Gwo, "Ultrafast hot electron relaxation time anomaly in InN epitaxial film," Appl. Phys. Lett. 90, 252111 (2007) 
S.-Z. Sun, Y.-C. Wen, S.-H. Guo, H.-M. Lee, S. Gwo, and C.-K. Sun, "Observation of femtosecond carrier thermalization time in indium nitride," J. Apply. Phys. 103, 123513 (2008).

[10] R. Ascazubi, I. Wilke, S. Cho, H. Lu, and W. J. Schaff, "Ultrafast recombination in Si-doped InN," Appl. Phys. Lett. 88, 112111 (2006).

[11] S. Nargelas, R. Alecksiejunas, M. Vengris, T. Malinauskas, K. Jarasiunas, and E. Dimakis, "Dynamics of free carrier absorption of InN layers," Appl. Phys. Lett. 95, 162103 (2009).

${ }^{[12]}$ K. Fukunaga, M. Hashimoto, H. Kunugita, J. Kamimura, A. Kikuchi, K. Kishino, and K. Ema, "Energy and density dependent dynamics of photoexcited carrier in InN films," Appl. Phys. Lett. 95, 232114 (2009).

[13] Y.-M. Chang and S. Gwo, "Carrier and phonon dynamics of wurtzite InN nanorods," Appl. Phys. Lett. 94, 071911 (2009).

[14] A. Othonos, M. Zervos, and M. Pervolaraki, "Ultra fast carrier relaxation of InN nanowires grown by reactive vapor transport," Nanoscale Research Lett. 4, 122 (2009).

[15] M. Fujiwara, Y. Ishihiro, X. Wang, S.-B. Che, and A. Yoshikawa, "Infrared analysis of hole properties of Mg-doped p-type InN films," Appl. Phys. Lett. 93, 231903 (2008).

[16] J. W. Ager III, R. E. Jones, D. M. Yamaguchi, K. M. Yu, W. Walukiewicz, S. X. Li, E. E. Haller, H. Lu, and W. J. Schaff, " $p$-type InN and In-rich InGaN," phys. stat. Sol. (b) 244, 1820 (2007).

[17] H. Ahn, Y.-P. Ku, C.-H. Chuang, C.-L. Pan, H.-W. Lin, Y.-L. Hong, and S. Gwo, "Terahertz spectroscopic study of vertically aligned InN nanorods," Appl. Phys. Lett. 91, 163105 (2007).

${ }^{[18]}$ H. Ahn, Y.-P. Ku, C.-H. Chuang, C.-L. Pan, H.-W. Lin, Y.-L. Hong, and S. Gwo, "Terahertz emission from vertically aligned InN nanorod arrays," Appl. Phys. Lett. 91, 132108 (2007).

[19] H. Ahn, C.-L. Pan, and S. Gwo, "Terahertz emission and spectroscopy on InN epilayer and nanostructure," Proc. of SPIE, 7216, 72160T (2009).

[20] H. Ahn, C.-H. Chuang, Y.-P. Ku, and C.-L. Pan, "Free carrier dynamics of InN nanorods investigated by timeresolved terahertz spectroscopy,” J. Appl. Phys. 105, 023707 (2009). 